\title{
Tables for Use in Quadrature Formulas Involving Derivatives of the Integrand
}

\section{By George Struble}

1. Introduction. Tables of the $a_{j}$ and $x_{j}$ in the approximate quadrature formulas developed by Hammer and Wicke [1]

$$
\begin{aligned}
& \int_{-1}^{1} f(x) d x=2 \sum_{i=0}^{(k-1) / 2} \frac{f^{(2 i)}(0)}{(2 i+1) !}+\sum_{j=1}^{m} a_{j}\left[f^{(k)}\left(x_{j}\right)-f^{(k)}\left(-x_{j}\right)\right], k \text { odd } \\
& \int_{-1}^{1} f(x) d x=2 \sum_{i=0}^{(k-2) / 2} \frac{f^{(2 i)}(0)}{(2 i+1) !}+\sum_{j=1}^{m} a_{j}\left[f^{(k)}\left(x_{j}\right)+f^{(k)}\left(-x_{j}\right)\right], k \text { even }
\end{aligned}
$$

are given in table 2 for $k=1,2, m=1(1) 10$. Coefficients of related orthogonal polynomials are given in table 1 for $k=1,2, m=0(1) 11$. The remainder terms for formulas (1) and (2) are

$$
R_{k, m}=\left\{\begin{array}{l}
\frac{f^{(4 m+k+1)}(\eta)}{(4 m+1) !} C_{k, m}, k \text { odd } \\
\frac{f^{(4 m+k)}(\eta)}{(4 m) !} C_{k, m}, k \text { even. }
\end{array}\right.
$$

The $C_{k, m}$ are listed in table 3 , for the same $k$ and $m$ as in table 2.

2. Calculations. The polynomials $P_{m, k}(x)$ were generated by orthonormalization of the serfuence $1, x, \cdots, x^{m}$ with weight functions

$$
W_{k}(x)=\left\{\begin{array}{l}
\frac{(1-\sqrt{x})^{k}}{k !}, k \text { odd } \\
\frac{(1-\sqrt{x})^{k}}{k ! \sqrt{x}}, k \text { even }
\end{array}\right.
$$

on the interval $(0,1)$. Their coefficients were obtained as solutions to systems of linear equations. The $x_{j}$ are the square roots of the zeros. $r_{j}$ of $P_{m, k}(x)$. The numbers $a_{j}$ are determined by

$$
a_{j}=\left\{\begin{array}{l}
\frac{A_{m+1, k}}{2 x_{j} P_{m, k}^{\prime}\left(r_{j}\right) P_{m+1, k}\left(r_{j}\right)}, k \text { odd } \\
\frac{A_{m+1, k}}{2 P_{m k}^{\prime}\left(r_{j}\right) P_{m+1, k}\left(r_{j}\right)}, k \text { even }
\end{array}\right.
$$

where $A_{m, k}$ is the quotient of leading coefficients of $P_{m, k}$ and $P_{m-1, k}$. The values of $C_{k, m}$ for use in the remainder terms were derived from

$$
C_{k, m}=\left\{\begin{array}{l}
2\left[\frac{(4 m+1) !}{(4 m+k+2) !}-\sum_{j=1}^{m} a_{j} x_{j}^{4 m+1}\right], k \text { odd } \\
2\left[\frac{(4 m) !}{(4 m+k+1) !}-\sum_{j=1}^{m} a_{j} x_{j}^{4 m}\right], k \text { even, }
\end{array}\right.
$$

Received June 1, 1959; in revised form, August 18, 1959. The calculations and work were supported by the Wisconsin Alumni Research Foundation and the Office of Ordnance Research, U. S. Army. 
TABLE 1

$$
k=1
$$

\footnotetext{
$P_{0}=1.73205080756888$

$P_{1}=7.53370803500884 x-2.26011241050265$

$P_{2}=30.8998823741862 x^{2}-23.6620720883408 x+2.68435271590420$

$P_{3}=125.095675561490 x^{3}-156.543424946216 x^{2}+49.9511087256310 x-3.04662582692541$

$P_{4}=503.922243199951 x^{4}-878.568454201908 x^{3}+480.772834453839 x^{2}-87.7416492632$ $052 x+3.36803132480860$

$P_{5}=2025.03058739579 x^{5}-4532.89143041580 x^{4}+3554.73528181640 x^{3}-1152.7142191499$ $2 x^{2}+138.227721512070 x-3.65996507211686$

$P_{6}=8126.6021735502 x^{6}-22225.892307314 x^{5}+22794.375219445 x^{4}-108.31 .6814728 .54 x^{3}+$ $2371.4844408556 x^{2}-202.49633196674 x+3.9293516425271$

$P_{7}=32585.34917364 x^{7}-105329.1554367 x^{6}+133708.3559097 x^{5}-84369.53936860 x^{4}+$ $27562.57790493 x^{3}-4390.474862558 x^{2}+281.5519951626 x-4.180763651686$

$P_{8}=130585.72574 x^{8}-487145.05832 x^{7}+737945.40041 x^{6}-582772.09740 x^{5}+256072.42057$ $x^{4}-61782.594359 x^{3}+7521.3030046 x^{2}-376.33313306 x+4.4174068709$

$P_{9}=523121.399 x^{9}-2212241.35 x^{8}+3896351.95 x^{7}-3702822.37 x^{6}+2055853.62 x^{5}-$ $674493.559 x^{4}+125947.128 x^{3}-12137.5185 x^{2}+487.723679 x-4.63163193$

$P_{10}=2095022.5 x^{10}-9904599.4 x^{9}+19892582 . x^{8}-22124981 . x^{7}+14891824 . x^{6}-$ $6225087.3 x^{5}+1595661.1 x^{4}-238415.82 x^{3}+18678.058 x^{2}-616.56109 x+4.8552190$

$P_{11}=8388194 . x^{11}-43842170 . x^{10}+98911790 . x^{9}-126115400 \cdot x^{8}+99931000 . x^{7}-$ $50973540 . x^{6}+16770010 . x^{5}-3468084 . x^{4}+425177.7 x^{3}-27649.29 x^{2}+763.6075 x-$ 5.059312
}

$$
k=2
$$

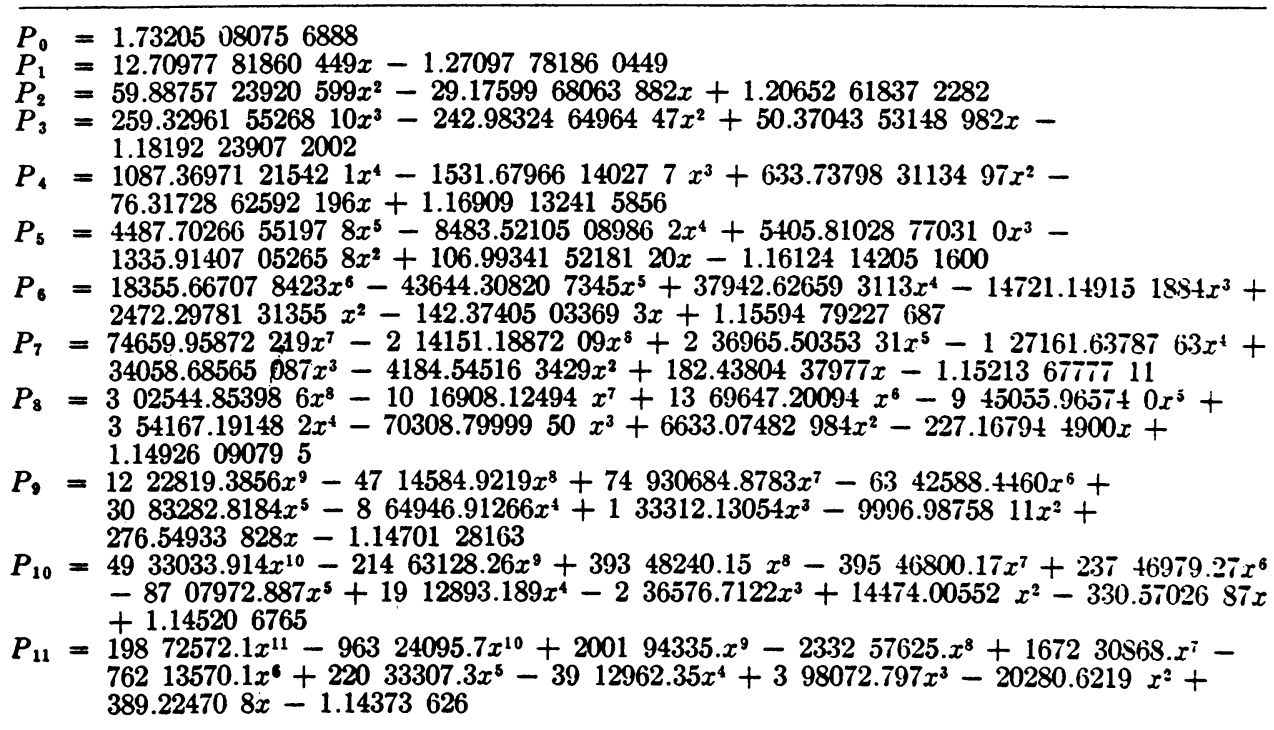


TABLE 2

\begin{tabular}{|c|c|c|c|}
\hline \multirow{2}{*}{$m$} & \multirow{2}{*}{$j$} & \multicolumn{2}{|c|}{$k=1$} \\
\hline & & $x_{j}$ & af \\
\hline 1 & 1 & .547722557505166 & .3042903097 .25092 \\
\hline 2 & $\begin{array}{l}1 \\
2\end{array}$ & $\begin{array}{l}.372145651138755 \\
.792005921760865\end{array}$ & $\begin{array}{l}.299869415177678 \\
.069534288047154\end{array}$ \\
\hline 3 & $\begin{array}{l}1 \\
2 \\
3\end{array}$ & 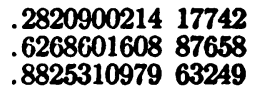 & $\begin{array}{l}.262516815542158 \\
.1158516504 \\
.0226513358 \\
.02470\end{array}$ \\
\hline 4 & $\begin{array}{l}1 \\
2 \\
3 \\
4\end{array}$ & 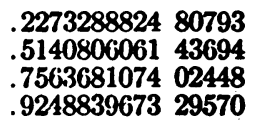 & $\begin{array}{l}.228302316462706 \\
.131369023471769 \\
.051020496676312 \\
.0093443207 \\
77566\end{array}$ \\
\hline 5 & $\begin{array}{l}1 \\
2 \\
3 \\
4 \\
5\end{array}$ & 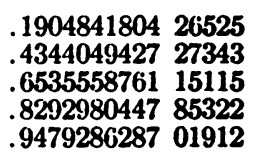 & 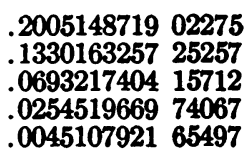 \\
\hline 6 & $\begin{array}{l}1 \\
2 \\
3 \\
4 \\
5 \\
6\end{array}$ & 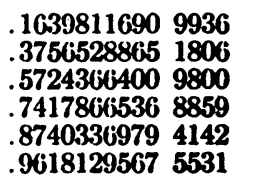 & $\begin{array}{r}.17821348158969 \\
.12904093879439 \\
.0790426562 \\
.0393058004 \\
.01950 \\
.0139874045 \quad 8116 \\
.00243240303689\end{array}$ \\
\hline 7 & $\begin{array}{l}1 \\
2 \\
3 \\
4 \\
5 \\
6 \\
7\end{array}$ & 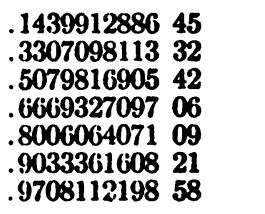 & 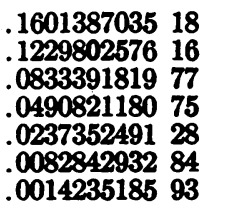 \\
\hline 8 & $\begin{array}{l}1 \\
2 \\
3 \\
4 \\
5 \\
6 \\
7 \\
8\end{array}$ & $\begin{array}{l}.1283703842 \\
.2952852684 \\
.4559661731 \\
.6038699343 \\
.7338622714 \\
.8415994425 \\
.9235314840 \\
.9769705854\end{array}$ & $\begin{array}{l}.1452769503 \\
.1163542537 \\
.0844453524 \\
.0553533943 \\
.0318100298 \\
.0151045544 \\
.0052043396 \\
.0008871299\end{array}$ \\
\hline 9 & $\begin{array}{l}1 \\
2 \\
3 \\
4 \\
5 \\
6 \\
7 \\
8 \\
9\end{array}$ & $\begin{array}{l}.115823631 \\
.2666673730 \\
.413291816 \\
.550684038 \\
.674964515 \\
.782771237 \\
.871235919 \\
.938022434 \\
.981369607\end{array}$ & $\begin{array}{l}.132878407 \\
.109816541 \\
.083725810 \\
.059056710 \\
.037887335 \\
.021412447 \\
.010039513 \\
.003428033 \\
.000581047\end{array}$ \\
\hline 10 & $\begin{array}{r}1 \\
2 \\
3 \\
4 \\
5 \\
6 \\
6 \\
7 \\
8 \\
9 \\
10\end{array}$ & $\begin{array}{l}.10552258 \\
.24309589 \\
.37773918 \\
.50552941 \\
.62345737 \\
.72888457 \\
.81949694 \\
.89332330 \\
.94876470 \\
.98461980\end{array}$ & $\begin{array}{l}.12239571 \\
.10363706 \\
.08198747 \\
.06098113 \\
.04220687 \\
.02667176 \\
.01489473 \\
.00691990 \\
.00234732 \\
.00039623\end{array}$ \\
\hline
\end{tabular}


TABLE 2-Continued

\begin{tabular}{|c|c|c|c|}
\hline \multirow{2}{*}{$m$} & \multirow{2}{*}{$j$} & \multicolumn{2}{|c|}{$k=2$} \\
\hline & & $x_{j}$ & $a_{j}$ \\
\hline 1 & 1 & .316227766016838 & .166666666666667 \\
\hline 2 & $\begin{array}{l}1 \\
2\end{array}$ & $\begin{array}{l}.213603621256443 \\
.6644945298 \\
23701\end{array}$ & 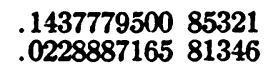 \\
\hline 3 & $\begin{array}{l}1 \\
\mathbf{2} \\
3\end{array}$ & 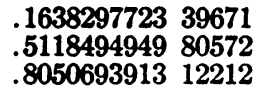 & $\begin{array}{l}.122479734491539 \\
.0395183540 \\
.0046685781 \\
64849\end{array}$ \\
\hline 4 & $\begin{array}{l}1 \\
2 \\
3 \\
4\end{array}$ & 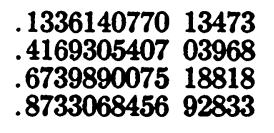 & 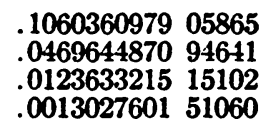 \\
\hline 5 & $\begin{array}{l}\mathbf{1} \\
\mathbf{2} \\
\mathbf{3} \\
\mathbf{4} \\
\mathbf{5}\end{array}$ & 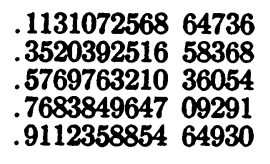 & 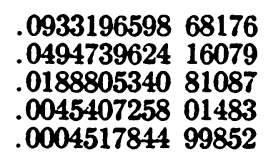 \\
\hline 6 & $\begin{array}{l}1 \\
2 \\
3 \\
4 \\
5 \\
6\end{array}$ & 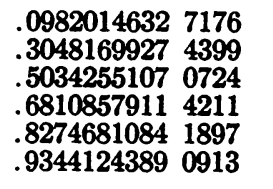 & 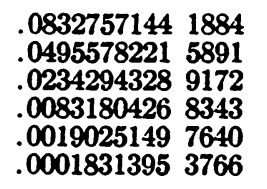 \\
\hline 7 & $\begin{array}{l}1 \\
2 \\
3 \\
4 \\
5 \\
6 \\
7\end{array}$ & 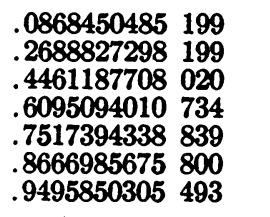 & 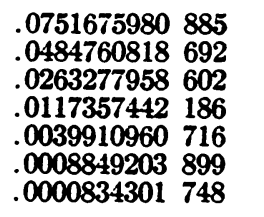 \\
\hline 8 & $\begin{array}{l}1 \\
2 \\
3 \\
4 \\
5 \\
6 \\
7 \\
8\end{array}$ & 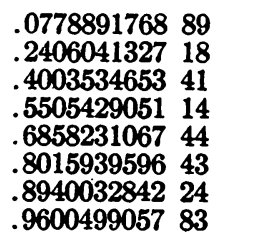 & 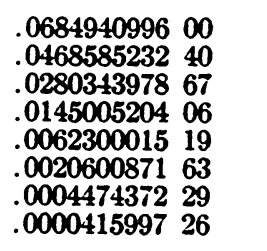 \\
\hline 9 & $\begin{array}{l}1 \\
\mathbf{2} \\
\mathbf{3} \\
\mathbf{4} \\
\mathbf{5} \\
\mathbf{6} \\
7 \\
\mathbf{8} \\
9\end{array}$ & 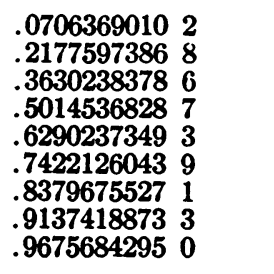 & 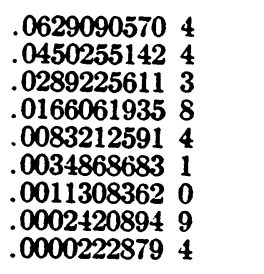 \\
\hline 10 & $\begin{array}{r}1 \\
2 \\
3 \\
4 \\
5 \\
6 \\
7 \\
8 \\
8 \\
9 \\
10\end{array}$ & $\begin{array}{l}.0646394150 \\
.1989137370 \\
.3320234405 \\
.4601078372 \\
.5800614933 \\
.6891397684 \\
.7849102517 \\
.8652666620 \\
.9284600004 \\
.9731500562\end{array}$ & $\begin{array}{l}.0581677528 \\
.0431420181 \\
.0292595373 \\
.0181452843 \\
.0101353888 \\
.0049671598 \\
.0020445253 \\
.0006538257 \\
.0001385086 \\
.0000126607\end{array}$ \\
\hline
\end{tabular}


TABLE $3^{*}$

\begin{tabular}{|c|c|c|c|}
\hline \multicolumn{2}{|r|}{$k=1$} & \multicolumn{2}{|r|}{$k=2$} \\
\hline$m$ & $C_{1, m}$ & $m$ & $C_{2 . m}$ \\
\hline $\begin{array}{r}1 \\
2 \\
3 \\
4 \\
5 \\
6 \\
7 \\
8 \\
9 \\
10\end{array}$ & $\begin{array}{l}1.7619 \times 10^{-2} \\
1.0473 \times 10^{-3} \\
6.3902 \times 10^{-5} \\
3.9380 \times 10^{-6} \\
2.4386 \times 10^{-7} \\
1.5142 \times 10^{-8} \\
9.4185 \times 10^{-10} \\
6.3112 \times 10^{-11} \\
7.7 \times 10^{-11} \\
-3.3 \times 10^{-10}\end{array}$ & $\begin{array}{r}1 \\
2 \\
3 \\
4 \\
5 \\
6 \\
7 \\
8 \\
9 \\
10\end{array}$ & $\begin{array}{r}6.1905 \times 10^{-3} \\
2.7882 \times 10^{-4} \\
1.4869 \times 10^{-5} \\
8.4576 \times 10^{-7} \\
4.9654 \times 10^{-8} \\
2.9680 \times 10^{-9} \\
1.7940 \times 10^{-10} \\
1.0922 \times 10^{-11} \\
6.8464 \times 10^{-18} \\
-2.8 \times 10^{-13}\end{array}$ \\
\hline
\end{tabular}

* These figures include round-off errors.

and thus show effects of round-off errors in $a_{j}$ and $x_{j}$ as well as the theoretical remainder. Since the matrix of the linear system solved to find the coefficients of $P_{m, k}(x)$ is ill-conditioned, the accuracy of the figures decreases rapidly with increasing $m$. Figures are kept to the upper limit of accuracy. If results are rounded to one or two fewer signific:ant figures than are carried in the table of $x_{j}$, there is no doubt of the ac:curacy of the digits kept.

The formulas were derived in [1]. The calculations were carried out in the Numerical Analysis Laboratory of the University of Wisconsin on the IBM 650 . An 18-digit floating-decimal interpretive routine by Eugene Albright and a linear systems solver by Gerald Thorne were used. I am indebted to William Kammerer for several helpful suggestions.

University of Wisconsin

Madison, Wisconsin

1. P. C. HAMmer \& H. H. WiCke, "Quadrature formulas involving derivatives of the integrand," Math. Comp. (MT'AC), v. 14, 1960, p. 3-7. 